\title{
A Computational Approach to Improve the Gathering of Ambient Assisted Living Requirements ${ }^{\dagger}$
}

\author{
Noelia García Castillo ${ }^{1,2, *}$, Juan Luis Pérez ${ }^{1}$ and Jorge J. Gómez-Sanz ${ }^{1}$ \\ 1 Dep. Software Engineering and Artificial Intelligence, Complutense University of Madrid, 28040 Madrid, \\ Spain; jlpdiez@ucm.es (J.L.P.); jjgomez@ucm.es (J.J.G.-S.) \\ 2 Conscious Management Institute (CMI), 28020 Madrid, Spain \\ * Correspondence: ngcastillo@pdi.ucm.es \\ + Presented at the 12th International Conference on Ubiquitous Computing and Ambient Intelligence \\ (UCAmI 2018), Punta Cana, Dominican Republic, 4-7 December 2018.
}

Published: 22 October 2018

\begin{abstract}
Recent publications focus on the importance of designing an Ambient Intelligence that can be sensitive to human values and responsible for its societal impact. Obtaining and properly modeling these requirements can be a challenging task. Co-creation and social sciences methods are frequently applied in order to discover what end-users need using methods such as field/case studies where interviews or focus group sessions are conducted. However, those methods can be limited. This paper introduces two complementary approaches, one using traditional semi-structured and in-depth interviews, and another one based on 3D simulation modeling. The context is a research project where interviews were conducted to caregivers of people with Alzheimer disease. When designing the solution, it is important to account what kind of technology the end-users are expecting and what scenarios need to be accounted. So, the paper first summarizes what technology this collective is seeking or willing to accept. Then it proceeds with a brief summary of one of the interviews. Following, it shows the process of transferring this information to actual 3D simulations and discusses the benefits of doing so in the context of Ambient Assisted Living.
\end{abstract}

Keywords: Ambient Intelligence; requirements

\section{Introduction}

Aging at home is a frequent desire shared by elderly people. It has not been proven that this preference changes in the case of dementia patients [1], so this desire could be granted by Ambient Intelligence (AmI) solutions providing patients and caregivers with a higher quality of life. This is called too Ambient Assisted Living (AAL), whose aim is to improve the quality of the daily living of individuals.

Nevertheless, to develop this AmI, it is required to elicit and represent human values, translate these values into technical requirements, develop the means to deal with moral dilemmas and values preferences, and to evaluate systems in terms of their contribution to human well-being considering the priorities of different stakeholders from diverse cultural context [2]. Human Computer Interaction (HCI) has addressed this problem before. The review of 102 works in mobile HCI [3] identified "a clear bias towards engineering systems using applied approaches" and, if there was an evaluation, this was made in a laboratory. Besides, researchers do not examine the phenomena in context such as case studies.

In the case of Alzheimer Disease (AD), there are little or none studies of what needs they have that can be covered with current AmI advances. People with Alzheimer are considered in general as part of a greater group, the older people, as in $[4,5]$. However, the Alzheimer is extended enough to be considered with more attention in the AAL domain. 
According to the World Alzheimer Report published in 2016, 47 million people are living with dementia worldwide, and, basing on prevalence rates, this number is projected to increase to more than 131 million by 2050 [6]. Caregivers and relatives of dementia patients undergo an 'evolving, increasingly challenging struggle to maintain continuity of roles, relationships, and lifestyles' [7].

Assistive Technology (AT) has been proven to be helpful in enabling and empowering individuals, in providing more safety and freedom of movements to people with dementia, in offering new activities and reassuring the patients, in reminding the person and in supporting orientation in time [8,9]. On the other hand, some of the benefits of applying AAL technologies to dementia care are described by caregivers as the peace of mind of the carers themselves [10] as well as improvements in other aspects of their well-being [9], but not necessarily the patients'.

Understanding better the implications of AT and its effects in patients becomes a key element in the development of AmI. The traditional means to address this involves social sciences methods (semi-structured interviews, focus groups, to cite some) and co-creation methods (open innovation, living labs). However, the degree of precision of human sciences methods to capture requirements cannot be precise enough for what engineers need. Also, the bias in traditional approaches in HCI [3] suggests that other ways need to be experimented.

The paper contributes to this debate with an simple requirements gathering case and its application to collect requirements in a Alzheimer assistance scenarios. The contribution is not yet a solution that increases their quality of daily living, but it helps in defining what problems there are and what expectations people have. The presented work illustrates how methods based in the $3 \mathrm{D}$ reconstruction of the daily living activities can be more helpful to identify more information that conventional social sciences methods, as in cultural heritage preservation [11].

The case study considers several interviews made within the project ColosAAL (TIN2014-57028-R) and focuses on a specific one where the relative discuses the utility of some AT device to assist the person outside the house. The interview also gathers information about situations the relative thinks are relevant, even if they are not necessarily related to the AT solution under study.

Using this as starting point, the research question is how much more information can be obtained if someone tried to actually model what happens in the house. The answer in this paper is a qualitative one. It shows which new information is discovered as a result. Creating those models requires effort, but making the model part of the development is a huge advantage. Also, this could help building that missing context in HCI that was identified by [3].

The models are built using the Ambient Intelligence Development Environment (AIDE) tool [12]. It is an open source tool that takes as input a visual description of scenarios and renders them into videos or 3D simulations. These 3D simulations can interact as well with emulated AmI devices, such as Android Emulators. Engineers can use these simulation to test their creations in the modeled scenarios.

This is interplay between simulations and AmI devices is an important one. Obtained models are not just for testing or evaluating the obtained information. After being coded, the models become the specification. They can be shared in different flavors, such as HTML documents, or the above-mentioned videos and simulations.

The paper is structured as follows. First the related work with AmI solutions for patients with Dementia in Section 2. Then, the methods used in this study are introduced in Section 3. The evaluation using social science methods and computer science are introduced in Section 4. These are discussed in Section 5. Afterwards, the conclusions are presented in Section 6.

\section{Related Work}

AT can help in the context of patients of Alzheimer to improve their live. The literature gathers different means tested to attain this goal.

Even though some research has been conducted, the role of AT in the domain of AD and related disorders is still debated, and the field would benefit from additional analyses' [8]. According to the existing literature, some of the examples of AAL technologies for dementia caregiving that are better 
accepted are tracking $[10,13,14]$, videoconferencing devices or technology mediated services $[9,14]$, fall alarms [13], voice interaction systems [15], and calendar clocks [13].

How useful is this assistance has been studied as well. Among the five factors that should be considered to assess the usefulness of assistive technology in the specific case of people with dementia, Hagen et al. [16] mentioned the impact of the family caregiver. The aim of this paper is to delve into this mentioned factor of acceptance and final use of assistive technology.

Landau et al. [10] conducted family caregivers' focus groups in Israel to discuss the use of tracking devices for dementia patients, some participants rejected to force the patient to use this technology, whereas most of those caregivers stated that the decision should be made by the family members who are in charge of the patient, even if he or she does not give consent.

Arntzen et al. [17] focused their study on the assessment of successful incorporation of AT for Norwegian people under 65 years of age who had recently been diagnosed with dementia, they found five requirements for AT incorporation in that context: addressing practical, emotional, and relational challenges; fit well into (...) established strategies; generate positive emotions; be user-friendly; and interest and engage the family carer as relatives have to strive for learning about technology and change their own established routines. This study also demonstrated the significant role that family caregivers play in whether or not, and in which ways, AT was absorbed into the everyday life of dementia patients. Finally, Arntzen et al. [17] concluded that the field needs to reveal the AT's positive as well as negative effects on $Y P D$ and family carer's everyday lives, and that of the utmost importance for a successful incorporation was the degree of engagement and interest of the family carer.

As a conclusion of their research, Evans et al. [18] confirmed that interviewing individuals with Dementia but also their caregivers will enable a user-centered design that will be essential for the development of assistive technology. In another way, understanding the requirements of the final users and increasing the acceptance of AAL technologies will not be possible. Moreover, this approach is essential to meet the standards of the person-centered care philosophy claimed by Kitwood [19] in the case of developing technological solutions that will affect their own lives. Nowadays, there is an increasing number of researches that involve the participation of relatives, such as the example of Coetzer [20] in designing person-centred assistive technologies.

According to Nauha et al. [13], those who would benefit from assistive technologies for people with memory impairment are home living elderly with mild dementia, even if further research is required to evaluate the utility of AT in different stages of a memory disorder and different life situations.

Topo [9] carried out a literature review of studies focused on the use of technology to support dementia's patients and their caregivers. He found that a low percentage of researches were considering patients living at home $-15 \%$ - whereas the rest studied the residential care of formal caregivers. As a result of this tendency in research, results are very much biased towards the residential care setting and toward moderate to severe stages of dementia. As a result of his comprehensive compilation, Topo [9] stated that an issue that needs urgent attention was assessing the perspective of those patients who still lived at home, by means of both qualitative and quantitative research, to investigate the potential of AAL in postponing residential care.

As a general conclusion shared by subsequent researchers $[9,15,17]$, they observed the conflicting needs and acceptance associated with the use of AT for this specific target depending on if the interviewed person was a patient or a family caregiver.

In general terms, dementia's patients tend to reject the installation of certain devices as they think that they are unnecessary and report their concern about sleep disorders, being active or being disoriented in time and space; however, a relative will consider patient's safety as a paramount issue. Holbø et al. [21] also referred to this dichotomy as the dementia patients that they consulted desired to maintain their lifestyle and the control over their own lives as well as their autonomy, reserving the intervention of family caregivers to safety-critical situations.

Other factors of influence related to the patients that were gathered by those researchers were the subjective experience of symptoms, their routines and skills, the characteristics of their local 
environment and the empathy for caregivers [21]. This last factor can be crucial to meet the requirements of both interested parties, as patients are sensitive of the safety concerns of their relatives. Kenigsberg et al. [8], affirm that these distant requirements for patients, relatives and professionals are related to a different understanding of the concept of risk that has evolved during the last decade. So, according to these researchers, understanding different ideas about risk is the first step in developing an effective risk mitigation strategy. Notwithstanding this general tendency of disagreement, Portet et al. [15] added a concern that was shared by both patients and caregivers in their experiment: the failure of the technological system.

\section{Methods}

The related work indicates that caregivers are crucial, and though AT may do little to slow down Alzheimer, it may help the caregivers. Only by means of a qualitative approach some concerns and perceptions expressed by patients and caregivers that have not been previously considered by researchers can be obtained. Due to that, we have conducted 20 open semi-structured interviews (i.e., without enforcing a particular order of questions to the interviewed) to delve into the explicit requirements of dementia's caregivers and on their technological attitudes. The findings obtained after analysing the semi-structured interviews with AtlasTi software will be discussed in light of the related work.

The population of the study are caregivers that were informed and agreed to a informed consent. The Sociodemographic variables describing the population are presented in Table 1 . The interviews had an average length of $60 \mathrm{~min}$. Principle caregivers of patients diagnosed with dementia, all living in the Community of Madrid (Spain), were questioned, in the following order, about the main challenges they face, about their own technological aptitudes and the ones of the patients, and about the technological solutions that they image that could be applied to mild dementia patients and their caregivers. Finally, we tested the concept of some AAL solutions that are being developed.

Table 1. Sociodemographic variables of dementia caregivers.

\begin{tabular}{lllllll}
\hline & Social class & Sex & Age & Level of studies & Occupation & Role \\
\hline C1 & Upper middle & Man & & Secondary & Receptionist & Son \\
C2 & Upper middle & Woman & 55 & University & Lecturer & Daughter \\
C3 & Middle & Women & 55 & University & Teacher & Niece \\
C4 & Middle & Man & 62 & University & Lecturer & Son-in-law \\
C5 & Upper middle & Woman & 62 & Primary & Homemaker & Daughter \\
C6 & Middle & Woman & 56 & University & Part time teacher & Daughter \\
C7 & Upper middle & Man & 78 & University & Retired computer & Husband \\
C8 & Middle & Woman & 39 & University & Graphic designer & Daughter \\
C9 & Middle & Woman & 59 & University & Pharmacist & Daughter \\
C10 & Middle & Man & 56 & Secondary & Bird breeder & Son \\
C11 & Lower middle & Woman & 60 & University & Architectural & Professional \\
C12 & Upper middle & Woman & 60 & University & Retired teacher & caregiver \\
C13 & Middle & Man & 58 & Secondary & Graphic designer & Son \\
C14 & Lower middle & Woman & 47 & Secondary & House cleaner & Daughter \\
C15 & Middle & Woman & 60 & Primary & Homemaker & Daughter-in-law \\
C16 & Middle & Woman & 65 & Primary & Homemaker & Daughter \\
C17 & Lower middle & Woman & 64 & Primary & Homemaker & Daughter \\
C18 & Upper middle & Woman & 26 & University & Pharmacist & Daughter \\
C19 & Middle & Man & 66 & University & Retired administrative & Husband \\
& & & & & assistant & Retired administrative \\
C20 & Middle & Woman & 56 & Secondary & assistant & Daughter-in-law \\
& & & & & &
\end{tabular}


To evaluate the incompleteness of these interviews, a posterior work of modeling with computer science methods was tackled. The approach was to create 3D computer simulations using AIDE facilities [12]. The modeling exercise is a necessary expensive task that allows to discover gaps in the interviews and allows to make new questions about the real needs of the patients.

\section{Results}

In this section, it can be compared the precision in the requirements gathering using a qualitative analysis of semi-structured interviews and the results obtained when translating this information in a $3 \mathrm{D}$ recreation of the situations. It is expected the second outperfoms the first in terms of level of detail and reusability along the development.

The section starts analyzing what caregivers expect (Section 4.1) after the recent technological advances. We use this as starting point before studying the quality of gathered information using social sciences methods (Section 4.2). Then, the 3D modeling based one is used in Section 4.3 so that both can be compared in Section 5 .

\subsection{What Caregivers Expect}

Before mentioning any specific solution, caregivers were asked to speak about examples of technology, existing or non-existing, that could be helpful for recently diagnosed or mild dementia patients and their caregivers. Initially, interviewees found difficult to imagine new technological proposals, but after a moment of reflection, most carers were able to find at least one example. It is remarkable that those technological solutions tend to be similar to technologies that are currently available or under development. Moreover, some of these spontaneous examples are those concepts of AAL solutions mentioned by the interviewer in the following question.

The commonest proposals are those related to indoor geolocation and home automation systems. As it is admitted by C11, this example is a mere modification of the available remote care services: wristbands that are worn by both patient and carer. C6 suggests a system that warns you if the patient is leaving his or her room and goes to the toilet. They state that this example will be useful for patients to avoid disorientation, but particularly for caregivers and relatives as they would be able to rest at night.

There is some agreement on the same example in which lights turn on and off automatically so patients do not need to look for the switch and the risk of destabilization and fall can be reduced. Some interviewees are worried about the inviolability of the home. Some improvements that would be added by caregivers to the system are preventing strangers from entering the house, the installation of gas leak and smoke sensors, switching off the doorbell and that an alarm notifies the caregiver if the main door is open, $\mathrm{C} 14$ uses the term domotic door (an door opening automatically). Those solutions are important during the first stage of the disease as, according to this carer, dementia patients open the door to anyone.

Other shared requirement is the improvement of the communication systems between patients and their relatives. Interviewees agree on their preference for videoconference instead of telephone. In some cases, patients get distressed if they are left alone at home because they do not remember where their relatives are and when they will be back, so the use of image and voice could calm them down better.

Other technological solutions adapted to their situation are digital calendars, using technology to reduce the intake of medicines in cases of anxiety or irritability-chromotherapy and music therapyor a device that keep patients active. Other proposal is a system that warns the patients if they are putting or looking for something out of its place: this element is not proper to be into the oven; and it would help patients to keep and order and to find things (C10) because not finding their belongings distresses dementia patients.

Regarding the technological interfaces that can be used, caregivers spontaneously mentioned pendants or wristbands for geolocation, and tablets or television sets for videoconferences. 


\subsection{A Specific Interview with $\mathrm{C} 3$}

As previous section showed, geolocation (indoor and outdoor) are important elements according to the caregivers. Automation would be welcome as well. The specific use of geo-location and automation should be discussed following depending on the circumstances of each caregiver and the patient.

With respect this discourse of other caregivers, C3 introduces some modifications: that the system can function outside the house, that it allows direct contact between patient and caregiver and that it is waterproof so it would not need to be removed during her activities of personal hygiene.

C3 finds this AT solution especially useful when the patient spends the summer alone in another town. She suspects something like this may exist already. The C3 interviewee proposes that such a locating device should be as small as possible so the patient could carry it everywhere on her own body like a little medal and that it should allow them to communicate. It should be noted that she uses the term little medal probably referring to the tele-care system, so her proposal could be read as a tele-care system with geolocation that works in exteriors and that allows direct contact with her main caregiver but not with an outside agent.

The geo-location is a recurrent topic, but knowing the situations and problems faced may help addressing how to use geo-location. To know more of this, some information of the person with Alzheimer is needed. The patient of this caregiver is an old female who was diagnosed as having an incipient senility 4 or 5 years ago, when she started noticing she sometimes forgot words and phone numbers. She lives alone but her two sisters and niece live close-by. Although she is capable of doing almost every day to day chore she is taken care by her small sister and niece who do almost everything for her. She is mostly aware of her illness and age and has more or less accepted she is close to death. This results in a better character and mood and giving less importance to small things. The problems they report she has are the following:

- Sometimes when speaking she forgets words. She is aware of that.

- Routinely forgets dates and times for schedules such as doctors.

- Forgets about tasks she has already done like having breakfast.

- She forgets about ever having been in places like the hospital where she usually goes.

- Has difficulties when she has to take decisions. For example at one time her house flooded and she didn't knew what to do about it.

- Has had mood changes. It is reported that before she was more grumpy but now she seems more calmed and relaxed.

- She has lower energy drive. Nowadays she is more lazy while before she was always doing things.

Having these issues, we address the problem of studying the different scenarios and guessing what uses geo-location may have in them.

\subsection{Modeling the C3 Case}

This section contains multiple diagrams using the AIDE tool. The diagrams are entity-relationship ones. A legend has been included into each one of them to facilitate their interpretation.

First, three symptoms has been modeled to capture the effect of Alzheimer into daily living activities. The symptoms are those presented in Figure 1. There are three of them: two related with memory loss (problems with speech and forgetfulness) and one with fatigue (mobility problems). In AIDE, the presence of a symptom can be low, medium, or high. In this case, we consider low values because we are at early stages of Alzheimer. 


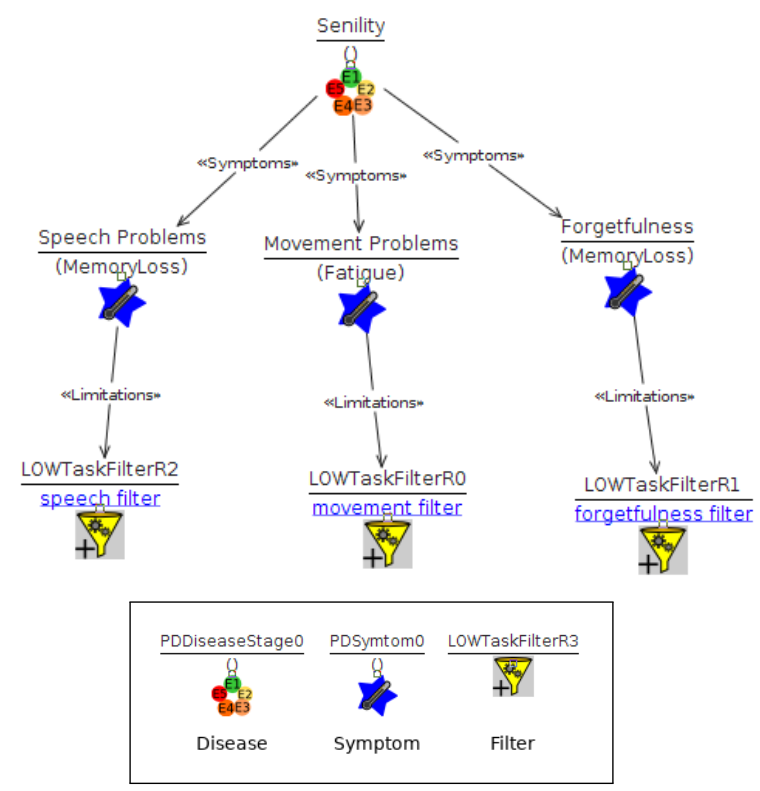

Figure 1. Modeling the symptoms.

Each symptom will be considered in two kinds of scenario: one involving activities of the daily living and another one more specific dealing with speech. Each scenario can be described in a positive way, determining what would be the sequence of events if there was no Alzheimer.

The positive sequence of activities is described into Figure 2. The figure assumes the patient goes to the bathroom, then uses the shower, following she uses the basin, then drinks water, then uses the toilet, and then goes to the kitchen, stopping next to the kitchen sink.

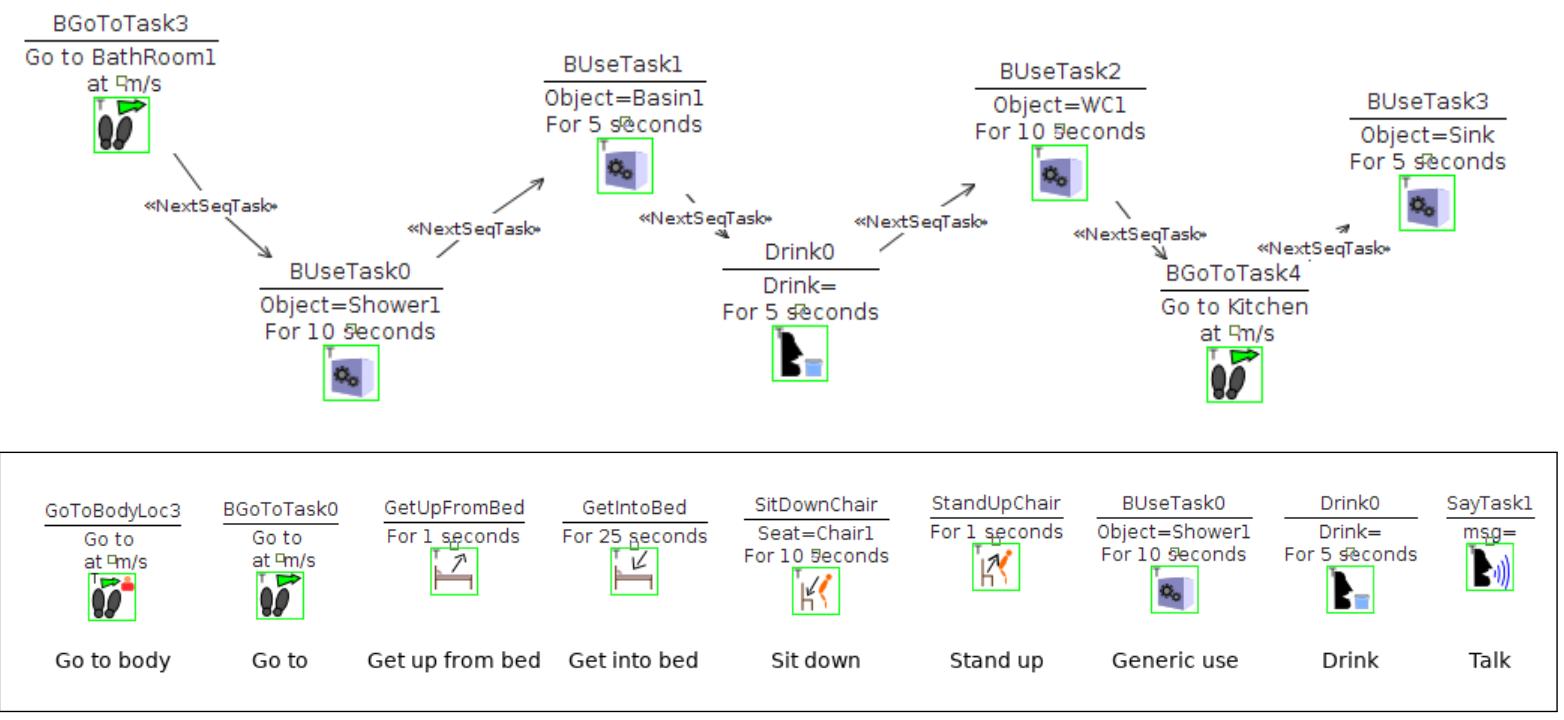

Figure 2. A sequence of tasks involving the bathroom and the kitchen.

Another sequence of activities is presented in Figure 3 and may model a nap. This time, it models a person that goes to the bedroom, lays down in the bed for a few minutes, then gets up, the goes to a chair, she sits down, and then stands up. 


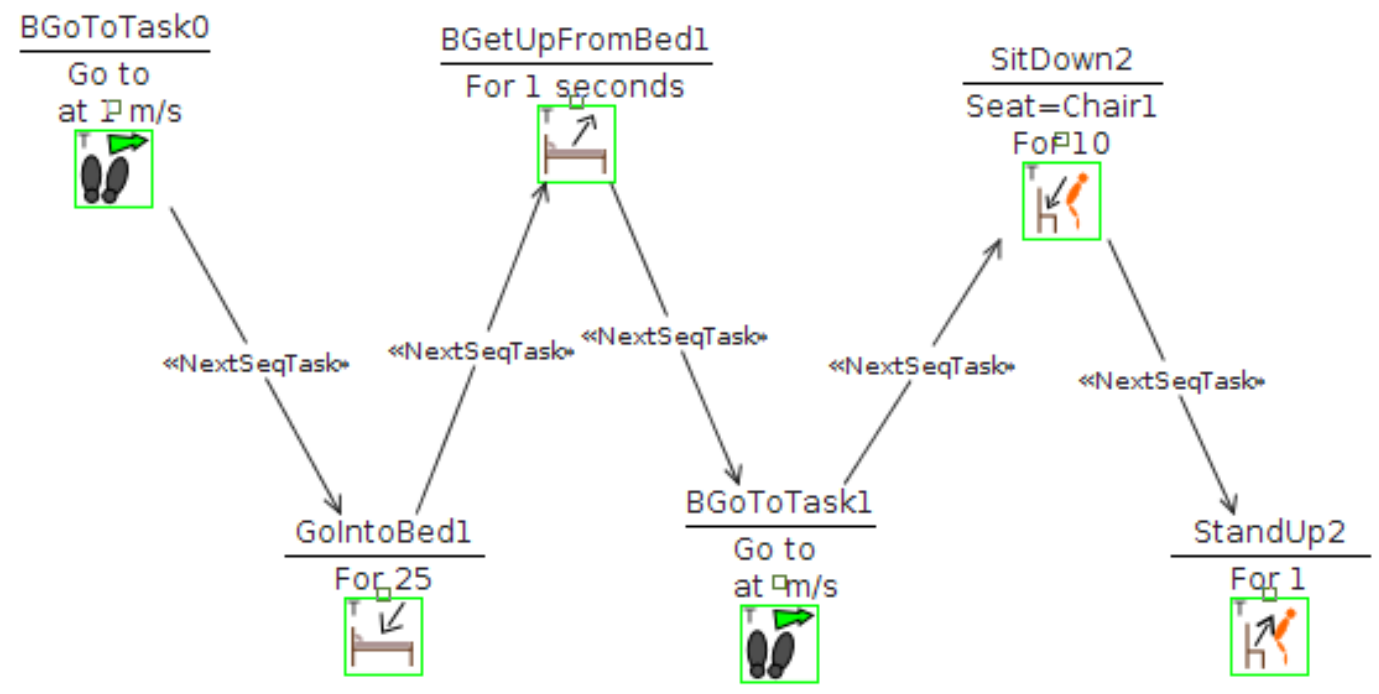

Figure 3. Sequence of moves the agent performs.

In the case of a dialogue, a sequence of utterances is performed. This time, it is harder to understand because it is modeled using events. The dialogue starts with actions on behalf the caregiver in Figure 4. The caregiver waits the person with Alzheimer to get close and starts a simple utterance (say Hello). The action finishes with the generation of an event NieceHelloFinish. The event is handled by the elements from Figure 5 shows what events are meaningful. Activities BActivity9, BActivity10, BActivity3, and BActivity4 point at sequences of tasks such as those of Figure 2, but including only those of type Talk, such as Figure 4. As a result, after each activity is finished, the simulator will translate text to a speech and will wait the utterance to finish. Following the example from Figure 4, the next action would be triggered would be BActivity 9 that makes the patient to answer the greeting and generate another event. This notation is not trivial to understand, but it allows to model events of different kinds in a homogeneous. Anyway, for the case of dialogues, it is already planned to include improvements.

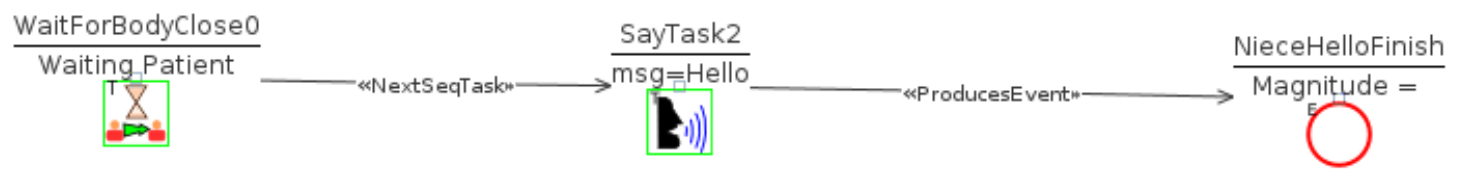

Figure 4. Initiating the dialogue. 


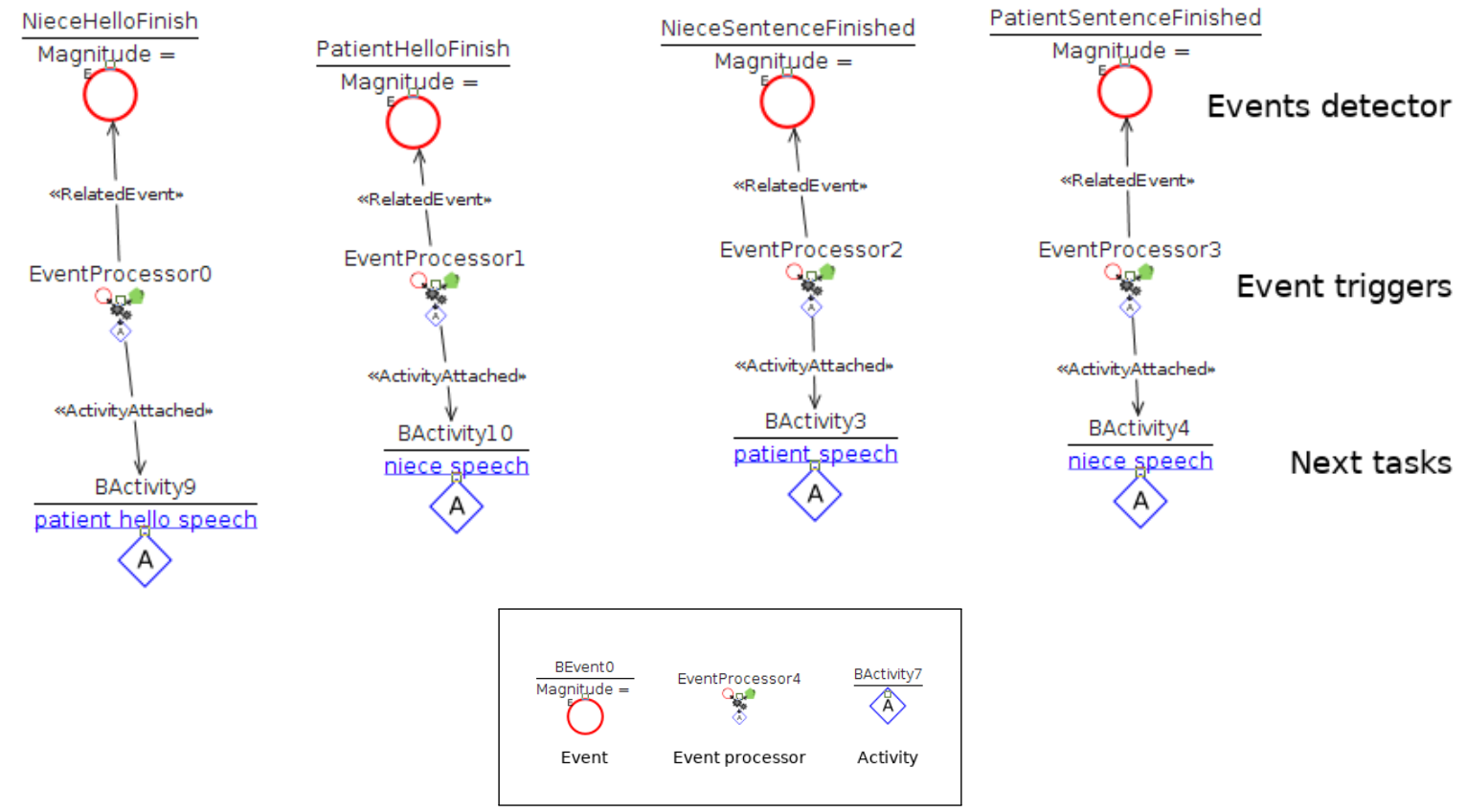

Figure 5. Representing the speech interaction.

The regular activities are modified by the existence of Alzheimer. In particular, we model three effects of the symptoms considered in Figure 1. The effects are captured using a Filter. This is a concept from AIDE that replaces or modifies one or many activities. The idea is to enable the modeler to express how a symptom modifies existing tasks. The alterations can be determined if a context for the activities is narrowed down. As an example, three scenarios have been extracted from the interview $\mathrm{C} 3$ and presented following:

- Scen1-Patient sometimes forgets a task she was about to perform. For this simulation a sequence of tasks was constructed that tries to mimic typical actions performed in the morning such as showering or drinking water. To model this, a filter is then applied that cancels some of the tasks that have to be performed. The movements the agent makes are presented in Figure 2 and they are affected by filters defined in Figure 6.

- Scen2-Patient is described as having a lower energy drive. In this case a simple sequence of getting in and out of bed, moving and using a chair was made. A filter is then applied that makes these actions take a longer time. The sequence of movements the agent performs is presented in Figure 3.

- Scen3-Patient sometimes forgets words. For this case we modeled a conversation and made a filter that makes her unable to speak on occasion. In this case, we try to model a conversation between the caregiver and the patient. See Figure 5.

For Scen1, the filtering to apply when forgetfulness happens (see Figure 1) is represented by Figure 6 . The filter replaces the current task with a probability of $0.075 \%$ with an activity described into diagram patient difficulties deciding. The diagram contains a single action which is an utterance of $I$ don't know what I was doing. 


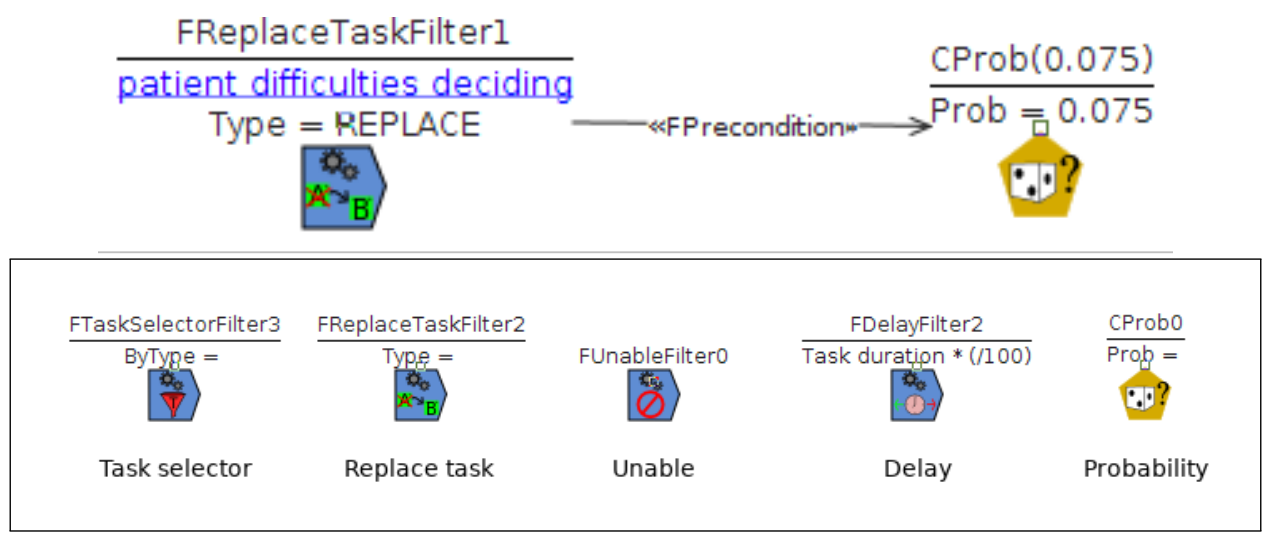

Figure 6. Forgetfulness symptom diagram.

For Scen 2 and lower drive, the symptom takes all tasks from Figure 3 and makes them slower. Figure 7 includes a filter that alters all tasks and makes them slowlier (50\%).

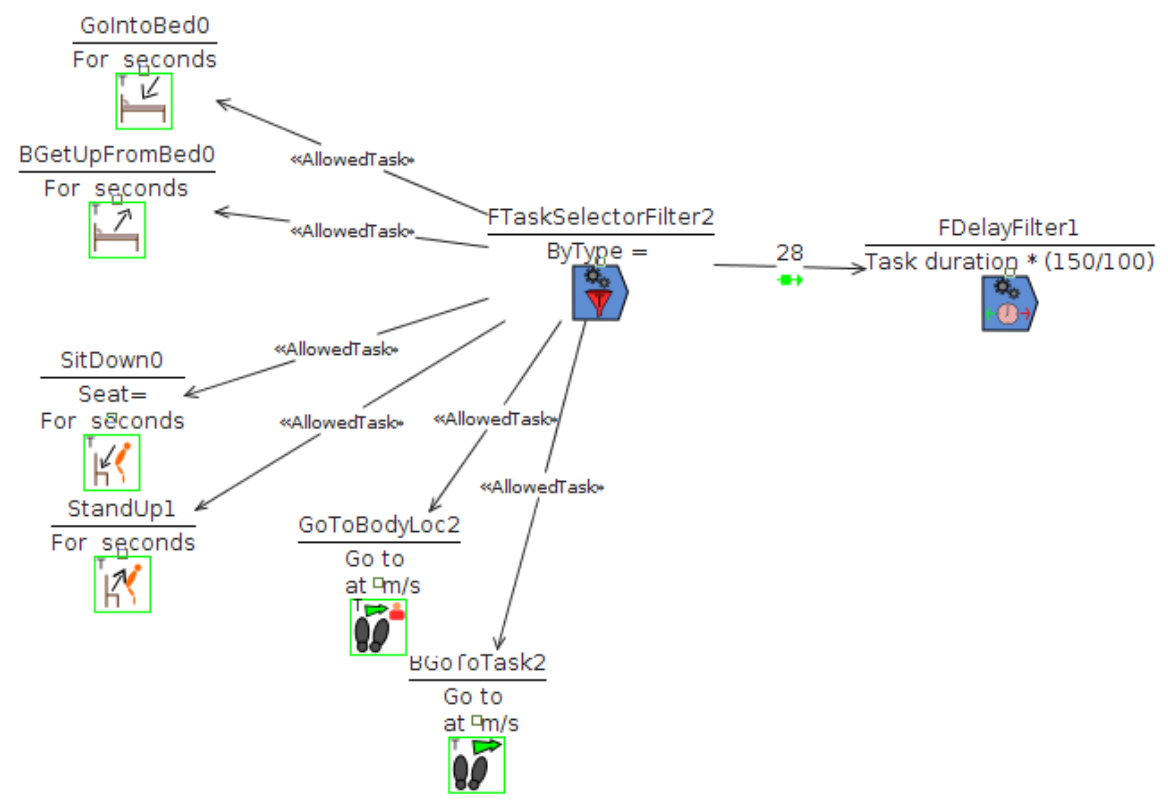

Figure 7. Filter applied to the movement problems simulation.

Lastly, to address Scen3, the modeling of this symptom was implemented using a filter of the unable type with a low probability $(0.075 \%)$ of this event happening (Figure 8$)$. The result of this activity will be to replace one of the utterances expected in Figure 5, interrupting this way the expected dialogue.

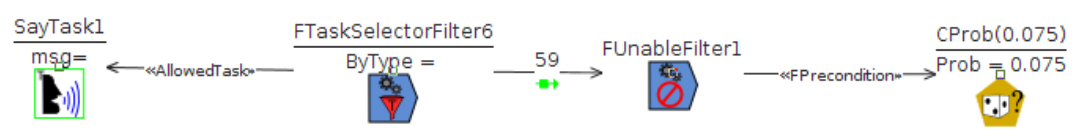

Figure 8. Filter applied to the speaking action.

The above mentioned positive activities and the effect of Alzheimer symptoms are included in a scenario specification like that of Figure 9. The scenario establishes an initial date and identifies participants. For each participant, their initial location is determined (Niece-Living Room, patient-Hall), 
and the patient is associated with a particular symptom. In this case, the symptom will enable the filters of memory loss declared in Figure 8. Each of these diagrams can contain different symptoms enabled at once.

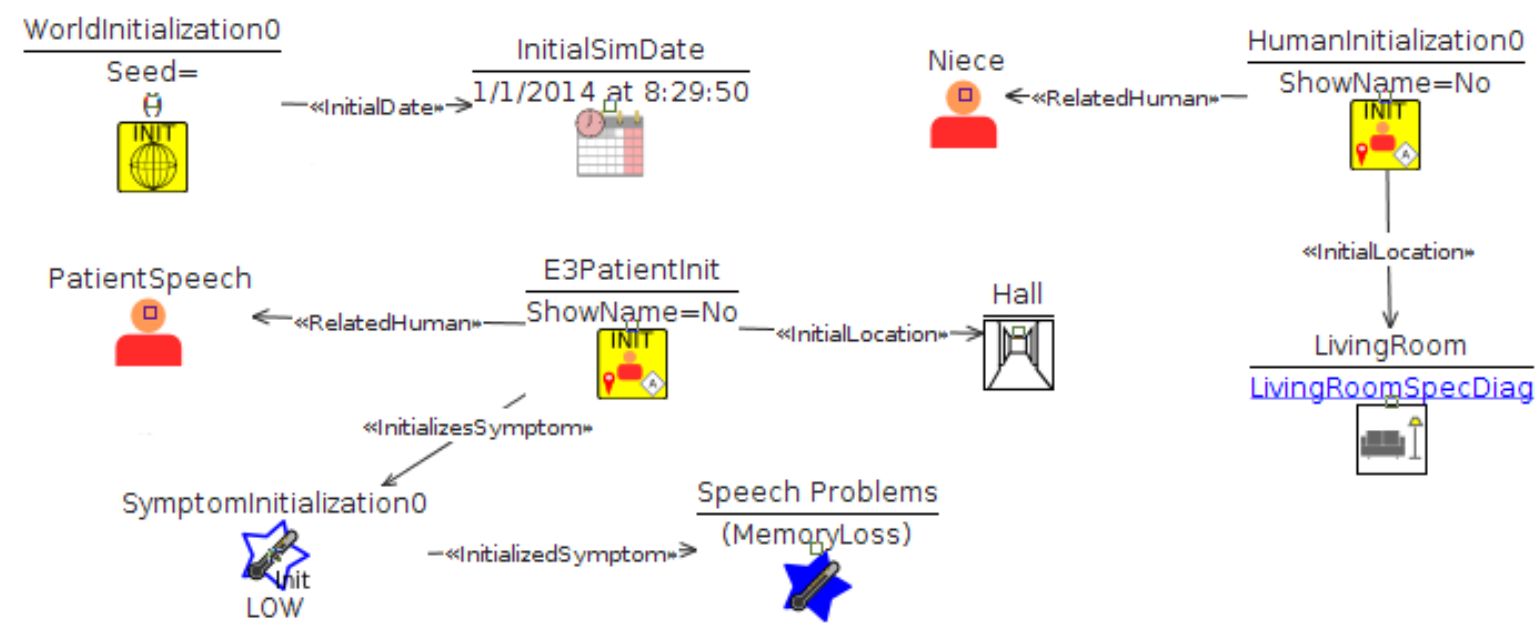

Figure 9. Representing the speech interaction.

After this point, the developer can initiate two :

- Discuss with other stakeholders to what extent the modeled scenarios capture the desired situations. Whether the positive sequence is what it should be and if these or additional effects are necessary. This leads to additional revisions of the specification and new simulations.

- Discuss what solutions could be applicable and evaluate the context each one will have to work on. The effect of the solution can be modeled as well and prototypes can be connected to the simulation. Restoring the without-symptoms status may not be possible, but it could be studied, from the perspective of the caregiver, what contingency actions would be accepted on behalf an AmI solution.

The rendering of definitions of scenarios such as Figure 9 leads to 3D simulations such as the one presented in Figure 10. The stakeholders can inspect the animation and evaluate the context. This is specially useful when there are multiple participants and different occurring actions. Showing them at the same time, helps stakeholders to understand better the problem.

Since the focus is on comparing both ways of addressing the problem, the next section will discuss a few issues (positive and negative) discovered during the modeling.

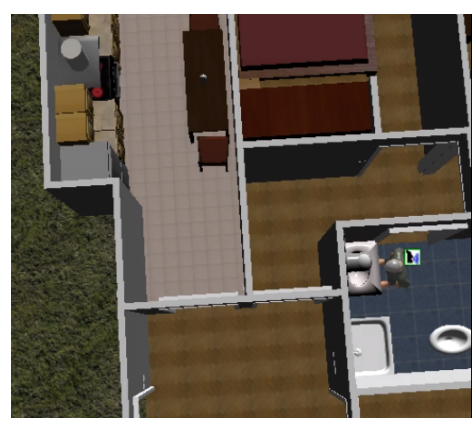

Figure 10. 3D simulation of the activities from Figure 2.

\section{Discussion}

Transforming a natural language description into an actual model necessarily involves discovering gaps and inconsistencies. In the case of these three scenarios, some issues were encountered. The main 
problem is that a lot of information gets lost along the way as there is no direct way of modeling things such as feelings, thoughts or emotions.

These affect the way human relationships develop, but, how they happen and which actions are affected was never accounted in the original interview. We guess that some of the activities could be altered by slowing them down even more or adding some additional pauses if the person with Alzheimer has emotional issues, such as depression.

Other statements are simply left for the developer to interpret. To give an example, there is no easy way of modeling the fact that she used to do a lot of stuff throughout the day whereas she is more "passive" nowadays. The statement could be forgotten, but it is relevant to address the passive part. Only by modeling positive sequences, such as those from Figures 2 and 3, it makes sense the passive part. We interpret that some actions are not initiated by the patient, but as a result of the initiative of the caregiver. Again, determining the context and the activities allow to understand better the interview.

About this context, it is not always described by the interviewee. We propose activities such as those presented in Figures 2 and 3. They can be combined with descriptions of the starting scenarios, such as Figure 1.

Of all the symptoms the patient and her caregiver describe many would get modeled similarly. For example in the case of forgetting already performed tasks or having difficulties while planning what to do next. In the modeling, these cases are modeled with a replacement of tasks (those in the positive sequence by others representing the negative side). The occurrence of the symptom is regulated by a random number generator, which makes one simulation run differently each time. This randomness is useful later on, when the solution is included in the analysis since the solution needs to distinguish when to intervene and when not. Sometimes, this randomness is not pursued. Such cases can be captured as well by making the replacement mandatory each time (happening with a probability of one).

Another difficult element to model would be in the case of losing oneself at home, which is closely related to the geo-location issue. Being lost at home is not the same as getting lost in a railroad station. Losing all the context and difficulties that would arise in one place or another makes the modeling less credible.

Another problem was the case of simulating a conversation where the patient forgets a word. The dialogue modeling was limited in terms of expressiveness (we already indicated this part is not easy to understand), and the allowed variations in the speech. Describing that a person cannot find the words to reply in a sentence is not possible at the moment. Instead, we model that one utterance is replace by other and that the expected event that continued the conversation is not produced anymore.

Despite these inconveniences, we find several potential advantages of this way of modeling with respect the qualitative analysis method:

- The higher precision when describing what happens, when, and where. The situation is different when a person falls in the same room the caregiver is, than when the caregiver is in a different room. Here, it may affect dialogue situations. Is it more difficult when both participants are in different rooms and sound is not reaching each one? AIDE permits to model this kind of situations and explore such situations.

- The benefits of developing the solution over the scenarios. Since there is a computational representation of what should happen (the positive sequence) and what should not happen (the resulting sequence after symptoms are enabled), it is possible to use these simulations as test bed. For instance, the solutions should be able, at least, to identify a negative sequence is in progress. A 3D simulation can produce a similar dataset to the one produced by a real environment, under controlled conditions [22].

- Better communication. Using plain text is risky to ensure the whole sequence is understood. AIDE allows to generate videos that can be commented and delivered to stakeholders. The videos are accompanied by diagrams (like those presented) and additional explanations in form of HTML pages. Also, we use this videos to start other co-creation activities, like focus groups. The videos 
can be used as motivating scenarios to trigger discussions. We expect this combination to be effective, but no experiments have been made to formally support this claim.

The effort of creating these models is greatly reduced by AIDE. The designer has to use a visual language to tell what the case study is about. AIDE renders the specification into visual animations that can be connected to emulations of AmI devices. The current example was part of a bigger set of interviews created during one month by an engineer that had no previous experience with AIDE. We have had worse experiences with people not having technical background. They can use the tool, but obtain worse results and it takes them longer to generate them.

\section{Conclusions and Further Research}

The paper has introduced a particular case study from a greater field study made within the project ColosSAAL and has shown how this information can be converted into computer models. The paper has introduced the particular case of one of the interviewees and tried to model some situations as described in the interview. This has produced a set of diagrams modeled with AIDE that represent a computational model that produces actual 3D simulations. A more exhaustive evaluation of the models and their effectiveness to communicate is pending, though.

It has helped discovering a number of problems and gaps in the specification, allowing to propose additional scenarios, each one filling in the gaps we found. As expected, the method is more robust and exhaustive than a pure qualitative method because specifications can actually be parsed and interpreted in terms of simulations.

Furthermore, by using AIDE, it allows to integrate the solution as part of the simulation and discuss what kinds of interplay with whom are allowed and/or desirable. Also, it makes the specification of the problem become part of the development, what reduces the chances of loosing information or developing something that is not related with the actual problem. On the cons side, a main problem is the high number of speculations needed in order to generate a context for the problems the caregivers enumerated. Fully defining the context is a necessary step towards defining the solution.

Another valid criticism is the scope of the current study which has been introduced. It only considers the patients' relatives point of view. This will bias any solution that affects patients of AD. Including them would imply even more serious ethical considerations and severe difficulties in accessing appropriate participants. Nevertheless, it is the only way to attain an inclusive design.

Funding: We acknowledge support from the project "Collaborative Ambient Assisted Living Design (ColoSAAL)" (TIN2014-57028-R) and "Collaborative Design for the Promotion of Well-Being in Inclusive Smart Cities" (TIN2017-88327-R) funded by Spanish Ministry for Economy and Competitiveness; also MOSI-AGIL-CM (S2013/ICE-3019) co-funded by Madrid Government, EU Structural Funds FSE, and FEDER.

Acknowledgments: To Millán Arroyo and Lucila Finkel for collaborating in the elaboration of the interview format.

Conflicts of Interest: The authors declare no conflict of interest.

\section{References}

1. Kinney, J.M.; Kart, C.S.; Murdoch, L.D.; Ziemba, T.F. Challenges in caregiving and creative solutions: Using technology to facilitate caring for a relative with dementia. Ageing Int. 2003, 28, 295-313.

2. Dignum, V. Responsible Artificial Intelligence: Designing Ai for Human Values. ITU J. ICT Discoveries 2017, $1,1-8$.

3. Kjeldskov, J.; Graham, C. A review of mobile HCI research methods. In International Conference on Mobile Human-Computer Interaction; Springer: Berlin, Germany, 2003; pp. 317-335.

4. Rashidi, P.; Mihailidis, A. A survey on ambient-assisted living tools for older adults. IEEE J. Biomed. Health Informat. 2013, 17, 579-590.

5. Memon, M.; Wagner, S.R.; Pedersen, C.F.; Beevi, F.H.A.; Hansen, F.O. Ambient assisted living healthcare frameworks, platforms, standards, and quality attributes. Sensors 2014, 14, 4312-4341. 
6. Prince, M.; Comas-Herrera, A.; Knapp, M.; Guerchet, M.; Karagiannidou, M. World Alzheimer Report 2016: Improving Healthcare for People Living with Dementia: Coverage, Quality and Costs Now and in the Future; Technical Report; Alzheimer's Disease International: London, UK, 2016.

7. Kart, C.; Kinney, J.; Murdoch, L.; Ziemba, T. Crossing the Digital Divide: Family Caregiver'S Acceptance of Technology; Scripps Gerontology Center, Miami University: Oxford, OH, USA, 2002.

8. Kenigsberg, P.A.; Aquino, J.P.; Bérard, A.; Brémond, F.; Charras, K.; Dening, T.; Droës, R.M.; Gzil, F.; Hicks, B.; Innes, A.; et al. Assistive technologies to address capabilities of people with dementia: From research to practice. Dementia 2017, 1471301217714093, doi:10.1177/1471301217714093.

9. Topo, P. Technology studies to meet the needs of people with dementia and their caregivers: A literature review. J. Appl. Gerontol. 2009, 28, 5-37.

10. Landau, R.; Auslander, G.K.; Werner, S.; Shoval, N.; Heinik, J. Families' and professional caregivers' views of using advanced technology to track people with dementia. Qual. Health Res. 2010, 20, 409-419.

11. Bruno, F.; Bruno, S.; De Sensi, G.; Luchi, M.L.; Mancuso, S.; Muzzupappa, M. From 3D reconstruction to virtual reality: A complete methodology for digital archaeological exhibition. J. Cult. Herit. 2010, 11, 42-49.

12. Campillo-Sánchez, P.; Gómez-Sanz, J.J. Modelling and Simulation of Alzheimer's Disease Scenarios. Procedia Comput. Sci. 2016, 83, 353-360, doi:10.1016/j.procs.2016.04.136.

13. Nauha, L.; Keränen, N.S.; Kangas, M.; Jämsä, T.; Reponen, J. Assistive technologies at home for people with a memory disorder. Dementia 2016, 1471301216674816, doi:10.1177/1471301216674816.

14. Rialle, V.; Ollivet, C.; Guigui, C.; Hervé, C. What do family caregivers of Alzheimer's disease patients desire in smart home technologies? Methods Inf. Med. 2008, 47, 63-69.

15. Portet, F.; Vacher, M.; Golanski, C.; Roux, C.; Meillon, B. Design and evaluation of a smart home voice interface for the elderly: Acceptability and objection aspects. Pers. Ubiquitous Comput. 2013, 17, 127-144.

16. Hagen, I.; Holthe, T.; Gilliard, J.; Topo, P.; Cahill, S.; Begley, E.; Jones, K.; Duff, P.; Macijauskiene, J.; Budraitiene, A.; et al. Development of a protocol for the assessment of assistive aids for people with dementia. Dementia 2004, 3, 281-296.

17. Arntzen, C.; Holthe, T.; Jentoft, R. Tracing the successful incorporation of assistive technology into everyday life for younger people with dementia and family carers. Dementia 2016, 15, 646-662.

18. Evans, J.; Brown, M.; Coughlan, T.; Lawson, G.; Craven, M.P. A systematic review of dementia focused assistive technology. In International Conference on Human-Computer Interaction; Springer: Berlin, Germany, 2015; pp. 406-417.

19. Kitwood, T.M.; Kitwood, T. Dementia Reconsidered: The Person Comes First; Open University Press: Buckingham, UK, 1997; Volume 20.

20. Coetzer, R. The potential role of relatives in designing person-centred assistive technologies (innovative practice). Dementia 2017, 1471301216688395, doi:10.1177/1471301216688395.

21. Holbø, K.; Bøthun, S.; Dahl, Y. Safe walking technology for people with dementia: What do they want? In Proceedings of the 15th International ACM SIGACCESS Conference on Computers and Accessibility, Bellevue, WA, USA, 2013; p. 21.

22. Gómez-Sanz, J.J.; Cardenas, M.; Pax, R.; Campillo, P. Building prototypes through 3D simulations. PAAMS 2016, 25, 299-301.

(c) 2018 by the authors. Licensee MDPI, Basel, Switzerland. This article is an open access article distributed under the terms and conditions of the Creative Commons Attribution (CC BY) license (http:// creativecommons.org/licenses/by/4.0/). 